\title{
A radial-velocity survey of Ap stars with HARPS ${ }^{\star}$ I. HD 42659: The discovery of the first spectroscopic binary
around a rapidly oscillating Ap star
}

\author{
M. Hartmann and A. P. Hatzes
}

\author{
Thüringer Landessternwarte Tautenburg, Sternwarte 5, 07778 Tautenburg, Germany \\ e-mail: michael@tls-tautenburg.de
}

Received 11 November 2014 / Accepted 14 August 2015

\begin{abstract}
Context. Chemically peculiar Ap stars are main-sequence stars of spectral type B8-F3 which show large overabundances of some chemical elements (e.g., $\mathrm{Si}, \mathrm{Sr}, \mathrm{Cr}$ ), especially of rare-earth elements, compared to normal A-type stars. Furthermore, they have strong, global magnetic fields and low rotational velocities. Some Ap stars exhibit high-overtone, low-degree, non-radial $p$-mode pulsations with periods of 6-24 min and are called rapidly oscillating Ap (roAp) stars. Interestingly, no roAp star is known to be a spectroscopic binary (SB), while many non-oscillating Ap (noAp) stars are found in SB systems.

Aims. The goal of this survey was to carry out a systematic search for sub-stellar and stellar companions around Ap stars.

Methods. Between 2004 and 2009, we observed 65 chemically peculiar stars with the HARPS spectrograph at the 3.6-m telescope at the European Southern Observatory (La Silla, Chile). The radial-velocity (RV) measurements were obtained using a new software called HARPS-TERRA which is based on a least-squares matching of each individual spectrum to a high signal-to-noise ratio template for each star.

Results. We find significant variability with a period of 93.2 days and a semi-amplitude of $11.85 \mathrm{~km} \mathrm{~s}^{-1}$ in the RVs of HD 42659 , a star of our sample that belongs to the group of roAp stars. This variability is caused by a stellar companion with a minimum mass of $0.47 M_{\odot}$ in a slightly eccentric $(e=0.146)$ orbit at a separation of $0.55 \mathrm{AU}$.

Conclusions. This detection makes HD 42659 the first confirmed SB around a roAp star in a relatively close orbit. It shows that tidal interactions in binaries do not necessarily inhibit pulsations in Ap stars.
\end{abstract}

Key words. techniques: radial velocities - binaries: spectroscopic - stars: chemically peculiar - stars: individual: HD 42659

\section{Introduction}

Chemically peculiar A (Ap) stars are main-sequence stars of spectral type B8-F3 which show large overabundances of some chemical elements (e.g., Si, Sr, Cr), especially of rare-earth elements. These abundances can be as high as 10000 times the solar values, or even higher. Furthermore, they possess strong, global magnetic fields of up to $34 \mathrm{kG}$. Compared to normal Atype stars, their rotational velocities are markedly lower; rotation periods typically range from several days to tens of years. As a result, spectra of Ap stars contain plenty of relatively narrow absorption lines, thus making these stars ideal targets for precise radial-velocity $(\mathrm{RV})$ measurements.

Some Ap stars exhibit high-overtone $(n \gg l)$, low-degree $(l \leq 3)$, non-radial $p$-mode pulsations with periods of 6-24 min and typical peak-to-peak $B$ amplitudes of up to $16 \mathrm{mmag}$. The longest pulsation periods were found recently in the stars HD 177765 (Alentiev et al. 2012, $23.6 \mathrm{~min}$ ) and HD 225914 (KIC 4768731; Smalley et al. 2015, $23.4 \mathrm{~min}$ ). A summary of these so-called rapidly oscillating Ap (roAp) stars can be found in Tables 1 of Kurtz et al. (2006) and Smalley et al. (2015).

Interestingly, according to the study by Hubrig et al. (2000), no roAp star is known to be a spectroscopic binary (SB). In contrast, many non-oscillating Ap (noAp) stars are detected in

* Based on observations collected at the European Southern Observatory (La Silla, Chile) under programs 074.C-0102, 075.C-0234, 076.C-0073, 079.C-0170, 080.C-0032, and 082.C-0308.
SB systems (Carrier et al. 2002). Moreover, other types of pulsating variables (e.g., $\beta$ Cep stars, $\delta$ Sct stars, or classical Cepheids) are often found in SB systems as well.

The explanation of this difference remains unclear up to the present. On the one hand, pulsations may be driven by tidal forces in close binaries (Cowling 1941; Zahn 1977), on the other hand, they might also be repressed or reduced due to tidal interaction (Hubrig et al. 2000). To prove the latter hypothesis, it is either necessary to show that all noAp stars are close binaries or that all roAp stars are single stars or wide visual binaries, as pointed out in Schöller et al. (2012).

Recently, Schöller et al. (2012) published their results of a multiplicity study of roAp stars. They investigated 28 roAp stars using near-infrared ( $K$-band) imaging with NAOS-CONICA (NACO) at the VLT. Overall, they detected 45 companion candidates around 13 of the observed stars, but their data consisted of only one epoch per star, making it hard to distinguish between gravitationally bound objects and chance projections. However, using a statistical approach, Schöller et al. (2012) were able to calculate a probability for the companion candidates to be unrelated to the primary source. Consequently, 39 of their candidates are very likely chance projections, but six systems might have a physical companion. These six candidates have angular separations, which range from 0.23 to $1^{\prime \prime} .78$, resulting in linear projected separations of 30-455 AU.

As already mentioned by Schöller et al. (2012), the three brightest roAp stars $\alpha$ Cir (HD 128898), $\beta$ CrB (HD 137909), 
Table 1. Stellar properties of HD 42659.

\begin{tabular}{llcc}
\hline \hline Parameter & & Value & References \\
\hline Spectral type & & A3 Sr Cr Eu & 1 \\
$V$ & {$[\mathrm{mag}]$} & $6.75 \pm 0.01$ & 2 \\
$M_{V}$ & {$[\mathrm{mag}]$} & $1.03 \pm 0.23$ & 3 \\
$\pi$ & {$[\mathrm{mas}]$} & $7.60 \pm 0.51$ & 4 \\
$d$ & {$[\mathrm{pc}]$} & $132 \pm 9$ & 4 \\
$\log T_{\text {eff }}$ & {$[\mathrm{K}]$} & $3.900 \pm 0.011$ & 3 \\
$\log L / L_{\odot}$ & & $1.48 \pm 0.09$ & 3 \\
$M_{1}$ & {$\left[M_{\odot}\right]$} & $2.10 \pm 0.10$ & 3 \\
$\log t$ & {$[\mathrm{yr}]$} & $8.88_{-0.03}^{+0.04}$ & 3 \\
$\tau$ & & $0.81_{-0.07}^{+0.06}$ & 3 \\
\hline
\end{tabular}

References. (1) Renson \& Manfroid (2009); (2) Høg et al. (2000); (3) Kochukhov \& Bagnulo (2006); (4) van Leeuwen (2007).

and $\gamma$ Equ (HD 201601) do have optical or physical companions, but these are in wide orbits. The companion of $\alpha$ Cir is listed as K5 V star with a separation of 15'.7 in the catalogue of Eggleton $\&$ Tokovinin (2008), while $\gamma$ Equ has three companions at distances of 1'.'0, 59!'4, and 335'. 8 according to the most recent data from the Washington Visual Double Star Catalog (Mason et al. 2012). The star $\beta$ CrB is a well-known binary. Neubauer (1944) derived the orbital elements from $341 \mathrm{RV}$ measurements taken at Lick Observatory between 1931 and 1942. Later, Oetken \& Orwert (1984) reinvestigated the spectroscopic orbit using 60 RV measurements obtained from spectrograms mainly taken at the Tautenburg Observatory between 1971 and 1983. They also determined the visual orbit using published speckle interferometric data.

\section{Stellar properties of HD 42659}

HD 42659 (HIP 29365, BD-15 1299, UV Lep) is classified as $\mathrm{A} 3 \mathrm{SrCrEu}$ star in the catalogue from Renson \& Manfroid (2009). Its visual magnitude is given as $V=6.75 \pm 0.01(\mathrm{H} \varnothing \mathrm{g}$ et al. 2000). Using the HIPPARCos parallax of $\pi=7.60 \pm$ 0.51 mas (van Leeuwen 2007), the star is located at a distance of $d=132 \pm 9 \mathrm{pc}$. The values of effective temperature, luminosity, mass, and absolute $(t)$ and fractional $(\tau)$ stellar age are taken from Kochukhov \& Bagnulo (2006) and shown in Table 1.

Rapid oscillations in this star were discovered by Martinez \& Kurtz (1994), who carried out photometric observations in 12 nights. They found oscillations with a 9.7-min period and a peak-to-peak Johnson $B$ amplitude of $0.8 \mathrm{mmag}$. Since this signal is not seen on some nights, the oscillations must be modulated in amplitude.

\section{Observations and data reduction}

In 2004, we started a survey to search for extrasolar planets and brown-dwarf companions around a sample of Ap stars using the HARPS $^{1}$ spectrograph at the 3.6-m telescope at the European Southern Observatory (La Silla, Chile). This spectrograph is a cross-dispersed échelle spectrograph with a resolving power $R=$ $\lambda / \Delta \lambda \approx 115000$. Each spectrum consists of 72 spectral orders covering the wavelength range 3780-5300 $\AA$ (order 161-116, blue CCD “Linda”) and 5330-6910 A (order 114-89, red CCD "Jasmin").

The primary goal of this program was to extend planet searches to stars more massive than the Sun, since these stars were mostly not considered by other planet search programs.

\footnotetext{
1 High-Accuracy Radial-velocity Planet Searcher.
}

However, some recent programs have been monitoring also more massive stars, such as evolved subgiants and giants (e.g., Hatzes et al. 2006; Döllinger et al. 2009; Niedzielski et al. 2009; Johnson et al. 2011; Sato et al. 2013) as well as A-F-type mainsequence stars (e.g., Galland et al. 2005; Guenther et al. 2009; Hartmann et al. 2010; Borgniet et al. 2014). One of our targets was the roAp star HD 42659.

Between 2004 and 2009, we acquired a total of 48 high signal-to-noise ratio $(\mathrm{S} / \mathrm{N})$ spectra in 16 nights during eight different observing runs with HARPS. Usually three consecutive spectra have been taken with a typical exposure time of $160 \mathrm{~s}$ each, yielding a median S/N of 100 at aperture 60 (order 100, central wavelength $6121 \AA$ ).

Before each observing night, the RV standard calibrations were executed. The data reduction was performed with an automatic pipeline, the HARPS Data Reduction Software (DRS). It included the standard reduction steps such as bias subtraction, flat-field correction, spectral order localization and extraction, and wavelength calibration.

\section{Radial-velocity measurements}

The RV measurements obtained with HARPS are normally derived using the cross-correlation function $(\mathrm{CCF})$ technique. This method uses a weighted binary mask of a certain spectral type. The reduced and calibrated stellar spectrum is cross-correlated with that mask and the RV, defined by the minimum of the CCF, is calculated by fitting a Gaussian function to the shape of the CCF. More details on this technique can be found in Queloz (1995) and Pepe et al. (2002). The RV determination, including a barycentric correction, is also implemented in the HARPS DRS. Since the earliest spectral type of the available binary masks is a G2 mask, this method is not optimal for RV measurements of Ap stars, although it seems to work quite well in some cases.

Therefore, we used a new software tool called HARPSTERRA (Template-Enhanced Radial velocity Re-analysis Application; Anglada-Escudé \& Butler 2012), which is based on a least-square template-matching approach. Initially, the highest $\mathrm{S} / \mathrm{N}$ observation is used as a preliminary template to obtain the $\mathrm{RV}$ for each spectrum. In the next step, a very high $\mathrm{S} / \mathrm{N}$ template is generated by co-adding all the spectra taking their individual RV shifts into account to calculate the RVs again. The entire procedure is applied for each échelle aperture individually and the final RV measurement is achieved by a weighted mean of the RVs of all apertures. For an extensive description of the algorithms, we refer the reader to Anglada-Escudé \& Butler (2012).

Table 2 lists all the differential RV measurements obtained for HD 42659. The corresponding time series is shown in Fig. 1. Obviously, it can be seen that the star is RV variable, and the scale of the variations gives clearly an indication of binarity.

\section{Period analysis and orbital solution}

Firstly, we analyzed the data by computing a periodogram in the range $1 \leq P \leq 10000$ days. Instead of the classical LombScargle periodogram (Lomb 1976; Scargle 1982), we used the generalized Lomb-Scargle periodogram (GLS; Zechmeister \& Kürster 2009). Here, measurement errors are taken into account and an offset is added as a free parameter to the sine-wave fitting. This approach seems to be more robust, since our data contain only a small number of independent measurements. The resulting periodogram is displayed in Fig. 2, which shows the highest power at a period of $P_{1}=93.04$ days $\left(f_{1}=0.010748 \mathrm{~d}^{-1}\right)$. We 
Table 2. Differential RV measurements for HD 42659.

\begin{tabular}{crr}
\hline $\begin{array}{c}\text { BJD } \\
(2400000+)\end{array}$ & \multicolumn{1}{c}{$\mathrm{dRV}$} & \multicolumn{1}{c}{$\sigma$} \\
{$\left[\mathrm{m} \mathrm{s}^{-1}\right]$} & {$\left[\mathrm{m} \mathrm{s}^{-1}\right]$} \\
\hline 53334.875495 & -496.5 & 36.5 \\
53334.877231 & -484.5 & 33.3 \\
53334.878979 & -521.6 & 38.5 \\
53463.481143 & -7352.7 & 119.1 \\
53463.482879 & -7299.3 & 122.8 \\
53463.484627 & -7400.2 & 122.6 \\
53464.485974 & -6532.0 & 168.1 \\
53464.487698 & -6597.4 & 157.4 \\
53464.489469 & -6528.1 & 155.3 \\
53581.916423 & 9269.6 & 196.3 \\
53581.918808 & 9383.6 & 191.9 \\
53581.920984 & 9355.7 & 183.2 \\
53582.908549 & 12094.0 & 282.9 \\
53582.910818 & 12224.8 & 251.9 \\
53582.913005 & 12116.4 & 236.1 \\
53582.916235 & 12221.9 & 230.9 \\
53711.683520 & -1896.8 & 163.1 \\
53711.686634 & -1834.3 & 163.5 \\
53711.689794 & -1885.9 & 159.9 \\
53713.636632 & -4117.2 & 160.3 \\
53713.640012 & -4030.9 & 174.9 \\
53713.643044 & -4171.3 & 151.8 \\
53715.606950 & -6348.9 & 135.7 \\
53715.609913 & -6464.1 & 123.9 \\
53715.613131 & -6254.1 & 109.8 \\
54222.520299 & 4967.0 & 223.8 \\
54222.522509 & 4898.0 & 222.5 \\
54222.524731 & 4890.9 & 232.6 \\
54224.512736 & 7428.1 & 144.2 \\
54224.514993 & 7412.2 & 136.8 \\
54224.517204 & 7423.0 & 141.1 \\
54338.924950 & 9836.4 & 154.6 \\
54338.927161 & 9869.4 & 151.1 \\
54338.929418 & 9916.4 & 147.4 \\
54443.634655 & 4649.9 & 193.6 \\
54443.636831 & 4667.2 & 193.7 \\
54443.639146 & 4554.4 & 204.8 \\
54864.757688 & -2335.2 & 214.2 \\
54864.759759 & -2243.0 & 207.5 \\
54866.674396 & -2953.5 & 132.2 \\
54866.676584 & -2731.8 & 140.6 \\
54866.678864 & -2702.8 & 144.9 \\
54868.523894 & 30.2 & 53.0 \\
54868.526070 & 0.0 & 48.7 \\
54868.528280 & 16.6 & 49.2 \\
54869.632330 & 653.1 & 91.9 \\
54869.634552 & 630.2 & 85.9 \\
54869.636774 & 633.7 & 86.8 \\
\hline & & \\
& &
\end{tabular}

derived a false alarm probability (FAP; probability that the detected signal would originate from noise) of less than $10^{-4}$ using a bootstrap randomization technique (Kürster et al. 1997).

There is a second peak visible in the periodogram exceeding the $1 \%$ FAP level at a period of $P_{2}=53.86$ days $\left(f_{2}=\right.$ $0.018567 \mathrm{~d}^{-1}$, FAP $\left.=0.0059\right)$. When subtracting the 93.04-day signal from the data, the 53.86-day signal disappears in the periodogram of the residuals and there is no significant additional signal. The same occurs when first subtracting the 53.86-day signal. Thus, we can conclude that one period is the alias of the other. This is further supported when looking at the window function of our observations. If we subtract both frequencies, i.e., $f_{2}-f_{1}=0.007819 \mathrm{~d}^{-1}$ (corresponding to a period of 127.89 days), we derive the period of a maximum in the window function (see peak at 127.58 days in bottom panel of Fig. 2).

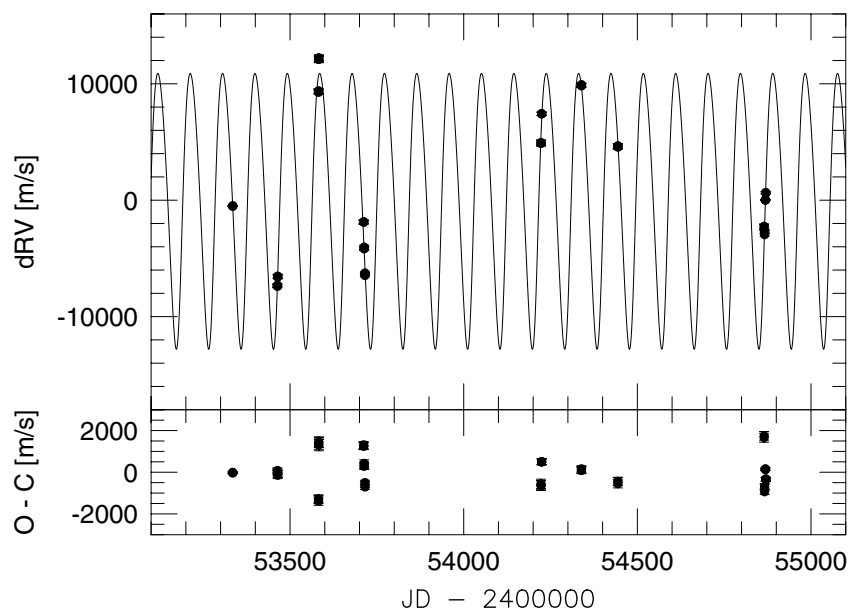

Fig. 1. RV measurements of HD 42659 and best-fit Keplerian orbital solution (solid line). The RV residuals after subtracting the orbital solution are shown below.

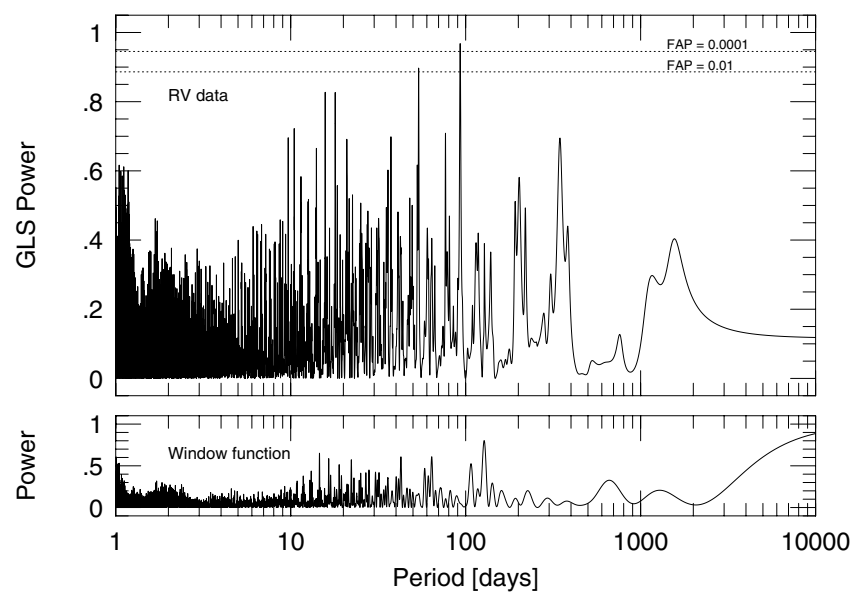

Fig. 2. Generalized Lomb-Scargle (GLS) periodogram of the RV data of HD 42659 (top panel). The highest peak is visible at a period of 93.04 days. The dotted lines indicate two levels of the false alarm probability (FAP). The bottom panel displays the window function of our observations.

Therefore, the data sampling does not allow us to distinguish which of the two periods is the true one, but the 93.04-day period is more likely.

Secondly, we fitted a Keplerian orbit to our measurements using the non-linear least-squares program GaussFit (Jefferys et al. 1988). The period we found ( $P=93.187 \pm 0.038$ days $)$ is fully consistent with the one obtained above. The orbit is slightly eccentric $(e=0.146 \pm 0.027)$ and also shown in Fig. 1 as well as in Fig. 3, where the data are phased to the orbital period. The phases were derived using the time of periastron passage $T_{0}=2454021.4$ as the zero point. The orbital parameters and theirs uncertainties determined by GaussFit from a maximumlikelihood estimation are given in Table 3. Using the calculated mass function, $f(m)=(15.6 \pm 1.7) \times 10^{-3} M_{\odot}$, and the primary stellar mass from Kochukhov \& Bagnulo (2006) (see Table 1) leads to a minimum companion mass of $0.468 \pm 0.021 M_{\odot}$ and a semi-major axis $a=0.551 \pm 0.008$ AU (Table 3 ).

\section{Discussion}

To our knowledge, HD 42659 is the first roAp star that is a spectroscopic binary. Although other roAp stars are known to be in binary systems, these are wide pairs with very long orbital 


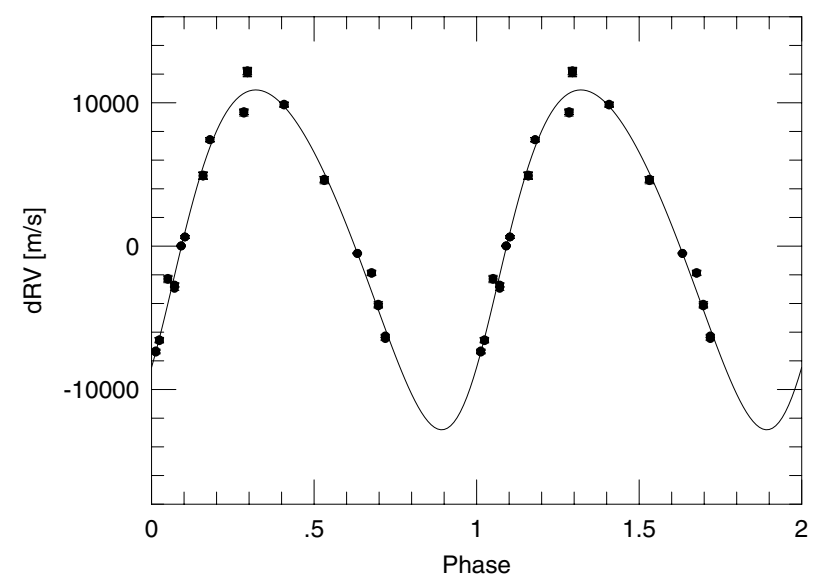

Fig. 3. RV measurements phased to the orbital period of 93.187 days. Phase zero corresponds to the time of periastron passage. The solid line represents the orbital solution. Data points are repeated for the second cycle.

periods. HD 42659 is the first roAp star in a relatively shortperiod binary system. Our derived orbital solution implies the presence of an early M-type main-sequence $\operatorname{star}\left(m=0.468 M_{\odot}\right)$ with a semi-major axis of $0.55 \mathrm{AU}(P=93.187 \mathrm{~d})$. We note that even if the true orbital period was the alias at 53.86 days, this would not affect our conclusion that there is a short-period binary companion to HD 42659 . In this case, the companion mass would be smaller.

Our discovery establishes that a lack of binarity is not a necessary condition for an Ap star to show rapid oscillations. In the case of HD 42659, the presence of the stellar companion seems to have no influence on the presence of oscillations. However, HD 42659 is one of the lowest-amplitude roAp stars, so it may be possible that the binary companion suppresses the oscillations, but not completely.

The binary orbit for HD 42659 has modest eccentricity and one may speculate that this may be responsible for the variable photometric amplitude seen in the photometric observations. As noted by Martinez \& Kurtz (1994), HD 42659 would have escaped detection as an roAp star if it were observed at the wrong time. It would be of interest to see if the times of low pulsational amplitude coincide to specific phases of the orbit, say periastron or apastron. This can only be answered with high-cadence photometric or spectroscopic (RV) time series at the appropriate binary orbital phases.

HD 42659 shows some similarities with the roAp star $10 \mathrm{Aql}$, an roAp star that is also difficult to detect. This star also has one of the lowest-amplitude photometric variations for an roAp star, with a semi-amplitude $\Delta B=0.44$ mmag (Heller \& Kramer 1990). In a spectroscopic study using precise RV measurements, Hatzes \& Mkrtichian (2005) found no significant variations in "integrated" RV measurements (i.e., using the full spectral range). However, stellar oscillations were clearly detected in only five spectral lines. Based on what spectral regions are examined, this star would either be an roAp star or not.

These selected spectral lines also showed amplitude variations when on some nights the oscillations disappeared entirely, similar to what is seen in the photometry of HD 42659. It is not known if $10 \mathrm{Aql}$ is also a short-period binary system. Hatzes \& Mkrtichian (2005) presented only three nights of RV data. Furthermore, this study only focused on the short-period oscillations and thus used only nightly relative velocities. A more detailed study is needed to establish if $10 \mathrm{Aql}$ is also in a binary system.
Table 3. Orbital elements of the companion of HD 42659.

\begin{tabular}{llc}
\hline \hline Parameter & & Value \\
\hline$P$ & [days] & $93.187 \pm 0.038$ \\
$T_{0}$ & {$[\mathrm{BJD}-2400000]$} & $54021.4 \pm 2.6$ \\
$K$ & {$\left[\mathrm{~m} \mathrm{~s}^{-1}\right]$} & $11854 \pm 426$ \\
$e$ & & $0.146 \pm 0.027$ \\
$\omega$ & {$[\mathrm{deg}]$} & $231 \pm 10$ \\
$\sigma_{\mathrm{O}-\mathrm{C}}$ & {$\left[\mathrm{m} \mathrm{s}^{-1}\right]$} & 799.1 \\
\hline$a_{1} \sin i$ & {$\left[10^{-3} \mathrm{AU}\right]$} & $100.5 \pm 3.6$ \\
$f(m)$ & {$\left[10^{-3} M_{\odot}\right]$} & $15.6 \pm 1.7$ \\
$m_{1}$ & {$\left[M_{\odot}\right]$} & $2.10 \pm 0.10$ \\
$m_{2} \sin i$ & {$\left[M_{\odot}\right]$} & $0.468 \pm 0.021$ \\
$a$ & {$[\mathrm{AU}]$} & $0.551 \pm 0.008$ \\
\hline
\end{tabular}

Although we have found one case of an roAp star in a shortperiod binary system, it appears that binarity is still a relatively rare phenomenon among roAp stars. How rare requires a dedicated RV search for binarity among roAp stars. Such a study may help provide further clues as to the origin of the roAp phenomenon.

Acknowledgements. The authors acknowledge the support of grants HA 3279/3-2 and HA 3279/3-3 from the Deutsche Forschungsgemeinschaft (DFG). We wish to thank G. Anglada-Escudé for providing the software HARPS-TERRA. We also thank the anonymous referee for making helpful suggestions that improved this paper. This research has made use of the SIMBAD database, operated at CDS, Strasbourg, France.

\section{References}

Alentiev, D., Kochukhov, O., Ryabchikova, T., et al. 2012, MNRAS, 421, L82 Anglada-Escudé, G., \& Butler, R. P. 2012, ApJS, 200, 15

Borgniet, S., Boisse, I., Lagrange, A.-M., et al. 2014, A\&A, 561, A65

Carrier, F., North, P., Udry, S., \& Babel, J. 2002, A\&A, 394, 151

Cowling, T. G. 1941, MNRAS, 101, 367

Döllinger, M. P., Hatzes, A. P., Pasquini, L., Guenther, E. W., \& Hartmann, M. 2009, A\&A, 505, 1311

Eggleton, P. P., \& Tokovinin, A. A. 2008, MNRAS, 389, 869

Galland, F., Lagrange, A.-M., Udry, S., et al. 2005, A\&A, 443, 337

Guenther, E. W., Hartmann, M., Esposito, M., et al. 2009, A\&A, 507, 1659

Hartmann, M., Guenther, E. W., \& Hatzes, A. P. 2010, ApJ, 717, 348

Hatzes, A. P., \& Mkrtichian, D. E. 2005, A\&A, 430, 279

Hatzes, A. P., Cochran, W. D., Endl, M., et al. 2006, A\&A, 457, 335

Heller, C. H., \& Kramer, K. S. 1990, MNRAS, 244, 372

Høg, E., Fabricius, C., Makarov, V. V., et al. 2000, A\&A, 355, L27

Hubrig, S., Kharchenko, N., Mathys, G., \& North, P. 2000, A\&A, 355, 1031

Jefferys, W. H., Fitzpatrick, M. J., \& McArthur, B. E. 1988, Celest. Mech., 41, 39

Johnson, J. A., Clanton, C., Howard, A. W., et al. 2011, ApJS, 197, 26

Kochukhov, O., \& Bagnulo, S. 2006, A\&A, 450, 763

Kürster, M., Schmitt, J. H. M. M., Cutispoto, G., \& Dennerl, K. 1997, A\&A, 320,831

Kurtz, D. W., Elkin, V. G., Cunha, M. S., et al. 2006, MNRAS, 372, 286

Lomb, N. R. 1976, Ap\&SS, 39, 447

Martinez, P., \& Kurtz, D. W. 1994, MNRAS, 271, 118

Mason, B. D., Wycoff, G. L., Hartkopf, W. I., Douglass, G. G., \& Worley, C. E. 2012, VizieR Online Data Catalog: I/2026

Neubauer, F. J. 1944, ApJ, 99, 134

Niedzielski, A., Nowak, G., Adamów, M., \& Wolszczan, A. 2009, ApJ, 707, 768

Oetken, L., \& Orwert, R. 1984, Astron. Nachr., 305, 317

Pepe, F., Mayor, M., Galland, F., et al. 2002, A\&A, 388, 632

Queloz, D. 1995, in New Developments in Array Technology and Applications, eds. A. G. D. Philip, K. Janes, \& A. R. Upgren, IAU Symp., 167, 221

Renson, P., \& Manfroid, J. 2009, A\&A, 498, 961

Sato, B., Omiya, M., Harakawa, H., et al. 2013, PASJ, 65, 85

Scargle, J. D. 1982, ApJ, 263, 835

Schöller, M., Correia, S., Hubrig, S., \& Kurtz, D. W. 2012, A\&A, 545, A38

Smalley, B., Niemczura, E., Murphy, S. J., et al. 2015, MNRAS, 452, 3334

van Leeuwen, F. 2007, A\&A, 474, 653

Zahn, J.-P. 1977, A\&A, 57, 383

Zechmeister, M., \& Kürster, M. 2009, A\&A, 496, 577 